\title{
Recursive Estimation of Exponential Signals in Impulsive Noise Using M-estimation
}

\author{
Zhiguo Zhang and Shing-Chow Chan \\ Department of Electrical and Electronic Engineering \\ The University of Hong Kong, Pokfulam Road, Hong Kong \\ zgzhang@eee.hku.hk, scchan@eee.hku.hk
}

\begin{abstract}
This paper proposes a robust method for recursive estimating the frequencies and amplitudes of an exponential signal model under impulsive noise. Using the concept of $M$-estimation, a recursive algorithm based on the recursive least $M$-estimate $(R L M)$ is developed. Simulation results show that the proposed method performs better than that of the conventional least square method under impulsive noise environment. The algorithm also possesses low arithmetic complexity due to its recursive nature.
\end{abstract}

\section{INTRODUCTION}

The complex exponential signal model is a very useful signal model in array signal processing, radar scattering, and other applications. The undamped exponential signal is modeled as:

$$
y_{n}=\sum_{j=1}^{p} \alpha_{j} e^{i \omega_{j} n}+\varepsilon_{n}, \quad n=1, \cdots, N,
$$

where $y_{n}$ is the observations, $i=\sqrt{-1}, \omega_{j} \in[0,2 \pi)$, $j=1, \cdots, p$, are the unknown frequencies of the $\mathrm{p}$ exponential signals, and $\alpha_{j}, j=1, \cdots, p$, are their unknown complex amplitudes. $\varepsilon_{n}$ is the additive noise corrupting the observations. The problem of estimating the frequencies and amplitudes of the exponential signals from a finite set of observations contaminated with Gaussian white noise is a traditional one, which has received considerable attention. A number of useful methods such as the Prony's method [1], the matrix pencil method [2], the exact maximum likelihood method [3], etc, are now available. Since the additive noise is usually assumed to be Gaussian distributed, the least squares ( $L S)$ error criterion is frequently employed in traditional estimation methods. One disadvantage of the $L S$ approach is its sensitivity to non-Gaussian noise exhibiting impulsive characteristics. Such impulsive noise occurs either naturally or is generated by various man-made sources. If the noise statistics is known, then maximum likelihood estimation ( $M L E$ ) can be used to combat the adverse effect of the impulsive or non-Gaussian noise. Since it is usually difficult to estimate accurately the statistics of the impulsive noise, a simple and efficient approach is to minimize the sensitivity of the estimator assuming a worse case distribution of the additive noise. This gives rise to the $M$-estimator (the maximum likelihood-like estimator) originally proposed by Huber [4]. This has motivated Wu et al [5] to study the robust parameter estimation of exponential signals under impulsive noise using $M$-estimation. Other related work includes Bootstrap $M$-estimation of Wen et al [6]. Both methods estimate the signal parameters in batch processing using a block of observation data. In this paper, we consider the time-recursive estimation of the exponential signals under impulsive noise environment. This leads to lower arithmetic computational complexity required to solve for the polynomial coefficients as well as the signal amplitudes. In addition, the signal parameters are continuously computed so that it is possible to track the slowly varying parameters. More precisely, the $R L M$ algorithm in [8-10] is proposed to estimate for the polynomials coefficients in the Prony's method. The frequencies of the exponential signal can then be computed from the roots of this polynomial. Once the frequencies of the exponential signals are determined, the $R L M$ algorithm can be used again to estimate the amplitudes of the frequency components. For simplicity, we shall assume that $p$ is known and the frequencies are distinct, i.e. $\omega_{l} \neq \omega_{m}$ if $l \neq m$.

The paper is organized as follows: in Section 2, the basic exponential signal model and the Prony's method is introduced. Section 3 is devoted to the proposed recursive parameter estimation algorithm of exponential signals. Its extension to the recursive $M$-estimation using the $R L M$ algorithm is then introduced in Section 4. Simulation results and comparisons are described in Section 5, and finally, conclusions are drawn in Section 6.

\section{Prony's Method}

In Prony's method [1], $\alpha_{j}$ and $\omega_{j}$ can be determined by solving two sets of linear simultaneous equations and a polynomial roots finding. Starting with $2 p$ observations $\boldsymbol{y}^{\prime}=\left[y_{0}, y_{1}, \cdots y_{2 p-1}\right]^{T}$, which can be rewritten as:

$$
\boldsymbol{y}^{\prime}=\boldsymbol{V} \cdot \boldsymbol{a}
$$

where $\quad V=\left[\begin{array}{cccc}1 & 1 & \cdots & 1 \\ e^{i \omega_{1}} & e^{i \omega_{2}} & \cdots & e^{i \omega_{p}} \\ \vdots & \vdots & \ddots & \vdots \\ e^{i \omega_{1} \cdot(2 p-1)} & e^{i \omega_{2}-2(2 p-1)} & \cdots & e^{i \omega_{p}(2 p-1)}\end{array}\right], \quad$ and $\alpha=\left[\alpha_{1}, \alpha_{2}, \cdots, \alpha_{p}\right]^{T}$. By properly choosing a predictor matrix $\boldsymbol{B}^{r}$

such that:

$$
\begin{aligned}
\boldsymbol{B}^{r} \boldsymbol{y}^{\prime} & =0, \\
\boldsymbol{B}^{T} & =\left[\begin{array}{llllll}
b_{p} & \cdots & b_{1} & 1 & & 0 \\
& \ddots & & \ddots & \\
\boldsymbol{0} & & b_{p} & \cdots & b_{1} & 1
\end{array}\right] .
\end{aligned}
$$

By using $b_{j}$ as coefficients, the following predictor polynomial can be formed:

$$
B(z)=\sum_{n=0}^{p} b_{n} z^{-n}=\prod_{n=1}^{p}\left(1-z_{n} z^{-1}\right)=0,
$$


where $b_{0}=0$ and $z_{n}=e^{i \omega_{n}}$. In other words, the zeros $z_{n}$ 's of the $B(z)$ correspond to the $p$ sinusoidal frequencies $\omega_{n}$. The equation $B^{\gamma} y^{\prime}=0$ can be rearranged to give

$z=-Y b$

where $\quad \boldsymbol{z}=\left[y_{p}, \cdots, y_{2 p-1}\right]^{T}, \quad \boldsymbol{Y}=\left[\begin{array}{cccc}y_{0} & y_{1} & \cdots & y_{p-1} \\ y_{1} & y_{2} & \cdots & y_{p} \\ \vdots & \vdots & \ddots & \vdots \\ y_{p-1} & y_{p} & \cdots & y_{2 p-2}\end{array}\right]$,

$\boldsymbol{b}=\left[b_{p}, \cdots, b_{1}\right]^{T}$. Hence, $\boldsymbol{b}$ can be computed from the given observations. The procedure can be summarized as follows:

Step I: $\quad$ Construct equation (5) using observations and solve the equation for $\boldsymbol{b}$;

Step II: $\quad$ Solve the polynomial $B(z)$ for roots $z_{n}$, and obtain the frequencies $\omega_{j}$;

Step III: Using least squares method to solve equation (2) for the amplitudes $\alpha_{j}: \alpha=\left(V^{*} V^{\prime}\right)^{-1} V^{*} y^{\prime}$.

\section{Recursive Parameter Estimation of EXPONENTIAL SigNAL MODEL}

In Section II, we can see that the basic Prony's method is a batch-processing algorithm, where a batch of observation data is processed to obtain the frequency and amplitude estimates of the signal. Here, a time-recursive algorithm will be developed to reduce the arithmetic complexity and allow tracking of slowly varying signal.

In step I, we can construct a set of functions from (5):

$$
y_{n}=-\boldsymbol{Y}_{n}^{\top} \boldsymbol{b}, n=p+1, \cdots, N
$$

where $\boldsymbol{Y}_{n}=\left[y_{n-p}, \cdots, y_{n-1}\right]^{T}$. Equation (6) means that the observation datum at time $n$ can be predicted linearly from the previous $p$ data, and the linear relation is determined by the coefficient vector $\boldsymbol{b}$. Alternatively, $\boldsymbol{Y}_{n}$ can be viewed as the input signal vector, and $b$ as the filter coefficient vector in a linear prediction problem. A recursive least-squares algorithm $(R L S)$ can then be used to estimate $b$ recursively. More precisely, the estimate at the time instant $n, b_{n}$, can be obtained by minimizing the weighted sum of magnitude-squared errors:

$$
E_{L S . b}=\sum_{l=p}^{n} \lambda_{b}{ }^{n-1}\left|e_{b}(l, n)\right|^{2}, n=p+1, \cdots, N .
$$

where $e_{b}(l, n)=y_{1}-\hat{y}_{l . n}=y_{1}-\left(-\boldsymbol{V}_{n}^{\gamma} \boldsymbol{b}_{n}\right)$, and $\lambda_{b}$ is the positive forgetting factor in the range $0<\lambda_{b} \leq 1$. More details of the $R L S$ algorithm procedure can be found in [6].

At time instant $n$, after we calculate the coefficient vector $b_{n}$ using the RLS algorithm, the roots of the polynomial $B_{n}(z)$ will be solved to obtain the frequencies $\omega_{j, n}$. Substituting it into the signal model (1), we have the following equation relating $\alpha_{j}$ and the observations $y_{n}$.
By considering the frequency components $\boldsymbol{X}_{n}=\left[e^{i \omega_{1, n n}}, \cdots, e^{i \omega_{p, n^{n}}}\right]^{T}$ as the input signal vector and consider $\alpha$ as the coefficient vector, the RLS algorithm can also be applied to calculate recursively the amplitudes vector $\alpha_{n}$ at time $n$. This is obtained by minimizing the $L S$ error function:

$$
E_{L S . a}=\sum_{s=1}^{n} \lambda_{a}^{n-s}\left|e_{\alpha}(s, n)\right|^{2}, n=p+1, \cdots, N .
$$

where $e_{\alpha}(s, n)=y_{s}-\hat{y}_{s . n}=y_{s}-\left(X_{s}{ }^{T} \boldsymbol{\alpha}_{s}\right), \quad \boldsymbol{\alpha}_{n} \quad$ is the estimation vector at time instant $n$ and $\lambda_{a}$ is a positive forgetting factor between 0 and 1 . Therefore, the frequency and amplitude of the sinusoids can be estimated recursively by the $R L S$ algorithm.

As mentioned earlier, the $L S$ method is very sensitive to outliers such as impulsive noise and its performance will degrade significantly. To address this problem, we will employ the robust $M$-estimator instead of the $L S$ estimator.

\section{RECURSIVE $M$-ESTIMATION}

The term " $M$-estimation" refers to "maximum likelihood estimation" or "generalized maximum likelihood estimation". Instead of minimizing the least squares error criterion in (7) and (9), the $M$-estimator uses a less rapidly increasing function as the error criterion. In this paper, the traditional Huber function is employed:

$$
\rho_{\xi}(e)=\left\{\begin{array}{cc}
e^{2} / 2, & 0<|e|<\xi \\
\xi|e|-\xi^{2} / 2 & |e| \geq \xi
\end{array}\right.
$$

where $\xi$ is a constant threshold parameter. It can be seen that if the residual error $|e|$ is smaller than $\xi$, the function $\rho_{\xi}(\cdot)$ is quadratic. But if the error $|e|$ is larger than $\xi$, $\rho_{\xi}(\cdot)$ becomes a linear function so that outliers with large amplitude can be suppressed. If the probability density function of the error is known, say $p(e)$, then choosing $\rho_{\xi}(e)$ as $-\log (p(e))$ gives the maximum likelihood estimator. In practice, the distribution of $e$ is not exactly known, and $\rho_{\xi}(e)$ is either chosen as the worse case distribution or other functions that are capable of suppressing the outliners. The parameter $\xi$ plays the role of suppressing the impulsive noise and it should be estimated continuously. Generally, we can estimate $\xi$ by $\xi=k \sigma(e)$, where $k$ is chosen as 2.576 .

Using the Huber function $\rho_{\xi}(e)$ instead of the square function, (6) and (8) can be rewritten as give the error criteria for the $M$-estimator:

$$
\begin{aligned}
& E_{M, b}=\sum_{l=p}^{n} \lambda_{b}^{n-1} \rho_{\xi_{b}}\left(\left|e_{b}(l, n)\right|\right), n=p+1, \cdots, N . \\
& E_{M, a}=\sum_{s=1}^{n} \lambda_{a}^{n-s} \rho_{\xi_{a}}\left(\left|e_{\alpha}(s, n)\right|\right), n=p+1, \cdots, N .
\end{aligned}
$$

Similar to the $L S$ case, (10) and (11) can be solved recursively using the recursive Least $M$-estimation $(R L M)$ $[8,9,10]$. Note, because (10) and (11) is a system of nonlinear equation, and it should be solved using the iterative methods described in [4]. The $R L M$ algorithm 
uses a weighted least squares approach to approximate this iteration and combines it with the $R L S$ algorithm for online estimation. It has been shown to be more effective than the $R L S$ algorithm when the input signals and desired signals are corrupted by impulsive noise. Detailed of the $R L M$ algorithm can be found in $[8,9,10]$. By using the $R L M$ algorithm, the following recursive algorithm for estimating the frequency and amplitude of the exponential signal under impulsive noise is obtained:

Step I: Use $R L M$ to calculate $\boldsymbol{b}_{n}$.

At time $p$, initialize the algorithm by setting: $\boldsymbol{P}_{b}(p)=\delta_{b}^{2} \boldsymbol{I}, \delta_{b}$ is a small constant, $\boldsymbol{b}_{p}=\mathbf{0}$.

At time $n,(n=p+1, \cdots, N)$, compute

$\boldsymbol{Y}_{n}=\left[y_{n-p}, \cdots, y_{n-1}\right]^{T}$,

$\boldsymbol{K}_{b}(n)=\frac{\boldsymbol{P}_{b}(n-1)\left(-\boldsymbol{Y}_{n}\right)}{\lambda_{b}+\left(-\boldsymbol{Y}_{n}\right)^{*} \boldsymbol{P}_{b}(n-1)\left(-\boldsymbol{Y}_{n}\right)^{\prime}}$,

$e_{b}(n)=y_{n}-\hat{y}_{n}=y_{n}-\left(-\boldsymbol{Y}_{n}{ }^{T} \boldsymbol{b}_{n-1}\right)$,

$\boldsymbol{b}_{n}=\boldsymbol{b}_{n-1}+\boldsymbol{K}_{b}(n) e_{b}(n)$,

$\boldsymbol{P}_{b}(n)=\left(\boldsymbol{P}_{b}(n-1)-\boldsymbol{K}_{b}(n)\left(-\boldsymbol{Y}_{n}\right)^{\prime} \boldsymbol{P}_{b}(n-1)\right) / \hat{\lambda}_{b}$.

Step II: Solve the roots of the polynomial $B_{n}(z)$ with $b_{n}$ as coefficients to obtain the frequencies $\omega_{j, n}$.

Step III: Use RLM to calculate $\boldsymbol{\alpha}_{n}$ at time $n$. Initialize the algorithm by setting: $\boldsymbol{P}_{a}(0)=\delta_{a}{ }^{2} \boldsymbol{I}, \delta_{a}$ is a small constant, $\boldsymbol{\alpha}_{0}=0$. At time $n$, compute $\boldsymbol{X}_{n}=\left[e^{i \omega_{,, n} n}, \cdots, e^{i \omega_{p, n^{n}}}\right]^{T}$, $\boldsymbol{K}_{a}(n)=\frac{\boldsymbol{P}_{\alpha}(n-1) \boldsymbol{X}_{n}}{\lambda_{\alpha}+\boldsymbol{X}_{n}^{*} \boldsymbol{P}_{\alpha}(n-1) \boldsymbol{X}_{n}{ }^{\prime}}$,

$e_{a}(n)=y_{n}-\hat{y}_{n}=y_{n}-\left(\boldsymbol{X}_{n}{ }^{T} \boldsymbol{\alpha}_{n-1}\right)$, $\boldsymbol{\alpha}_{n}=\boldsymbol{\alpha}_{n-1}+\boldsymbol{K}_{a}(n) e_{a}(n)$,

$\boldsymbol{P}_{\alpha}(n)=\left(\boldsymbol{P}_{\alpha}(n-1)-\boldsymbol{K}_{a}(n) \boldsymbol{X}_{s}^{\prime} \boldsymbol{P}_{\alpha}(n-1)\right) / \lambda_{\alpha}$.

Other than the root finding process, the arithmetic complexity of the algorithm is order $O\left(p^{2}\right)$, which is very low. It can be seen from step $I$ that the input signal vector is $\boldsymbol{Y}_{n}=\left[y_{n-p}, \cdots, y_{n-1}\right]^{T}$. In other words, impulsive noise will appear in the input signal. Although we can detect the impulsive noise in $y_{n}$ from the residual error $e_{b}(n)$, it is better to remove these input outliers at the very beginning. A pre-filter based on linear prediction is used to detect and remove these outliers in input vector. More precisely, at time instant $n^{\prime}$, we get the following estimate of $y_{n}$ : $\hat{y}_{n}=\sum_{j=1}^{n} \hat{\alpha}_{j, n^{\prime}-1} e^{i i_{j, n^{\prime}-1} n}$, based on the frequencies $\hat{\omega}_{j, n^{\prime}-1}$ and amplitudes $\hat{\alpha}_{j, n^{\prime}-1}$ obtained from the $R L M$ algorithm. The estimation error at time instant $n^{\prime}$ is $e\left(n^{\prime}\right)=y_{n^{\prime}}-\hat{y}_{n^{\prime}}$, where $\hat{y}_{n}$, is the predictor of $y_{n}$, based on the current estimates of the signal frequencies and amplitudes. By robustly estimating the variance of $e\left(n^{\prime}\right)=y_{n^{\prime}}-\hat{y}_{n^{\prime}}$ as [7-9]

$$
\hat{\delta}_{e}^{2}(n)=\lambda_{e} \hat{\delta}_{e}^{2}(n-1)+c_{1}\left(1-\lambda_{e}\right) \operatorname{med}\left(A_{e}(n)\right),
$$

where $c_{1}=1.483\left(1+5 /\left(N_{w}-1\right)\right)$ is a finite sample correction factor, $\operatorname{med}(\cdot)$ stands for median operator, $A_{e}(n)=\left\{e^{2}(n), \ldots, e^{2}\left(n-N_{w}+1\right)\right\}, \lambda_{e}$ is the forgetting factor and $N_{w}$ is the length of the estimation window, we can detect the presence of the impulsive noise by comparing $e\left(n^{\prime}\right)=y_{n^{\prime}}-\hat{y}_{n^{\prime}}$ with a threshold $\zeta=\kappa \delta_{e}(n)$. If $e\left(n^{\prime}\right)$ is greater than $\zeta$, an impulse is said to be presence and $y_{n^{n}}$ will be replaced by $\hat{y}_{n^{n}}$, otherwise, it is left unchanged.

This constitutes a new input vector $\hat{\boldsymbol{Y}}_{n^{\prime}}$, which is more robust to impulse noise. Reasonable values for $N_{w}$ are 511 , while those of $\kappa$ are $1.96-2.576$, depending on the tradeoff between impulse noise suppression and signal distortion.

\section{EXPERIMENTAL RESULTS}

Computer simulation was carried out to evaluate the proposed algorithm. The signal model is assumed to consist of two frequency components $(p=2)$ with frequencies $\omega_{1}=0.5$ and $\omega_{2}=1.2$, and respective amplitudes $\alpha_{1}=1$ and $\alpha_{2}=1.5$. The mean squared error (MSE) criterion is used to evaluate the estimations of $y$, $\omega$ and $\alpha$. The signal is corrupted by an additive white Gaussian noise with a $S N R$ of $35 \mathrm{~dB}$ at time instant $n=0,2, \cdots, 500$. To visualize the effect of the impulsive noise, the positions of the impulse are fixed at time instants: [100, 250, and 350], and their amplitudes are [6.6144, -11.3227, and 5.8227], which follow a Gaussian distribution with a large variance 100 . Unless otherwise specified, the following parameters will be used in the experiments: forgetting factors $\lambda_{b}$ and $\lambda_{a}$ are 0.99 , the threshold $\xi$ in (9) is chosen as $\xi=2.576 \cdot \sigma(e), \delta_{b}$ in step $I$ and $\delta_{a}$ in step III are 10. The MSE results are obtained by averaging the MSE results over 100 independent runs.

From Fig 1, it can be seen clearly that the RLM algorithm is more robust than the $R L S$ algorithm to the impulsive outliers at time instants [100, 250, and 350]. On the other hand, we can see that the initial convergence of the RLS algorithm is slightly faster than the $R L M$ algorithm. It is because, during initial convergence of the $R L M$ algorithm, estimation errors with large amplitude will be encountered and the algorithm will consider some of these errors as impulsive noise. This will somehow slow down the convergence speed. Better convergence speed can be obtained, if an iterative re-weight least squares algorithm is used to solve for (11) and (12). The $R L M$ algorithm at each time instant $n$ can be viewed as a one-step approximation of this iterative algorithm. Next, the effect of using different threshold values $\xi$ is studied. The parameter $k$ in $\xi=k \sigma(e)$ are chosen as $1.2818,2.576$ and 3.091 respectively, and the results shown as Fig 2 . It can be seen that the performance of the RLM algorithm together with the parameter estimation method is robust to the impulsive noise within a wide range of threshold values and it is not very sensitive to the choice of threshold $\xi$. In general, the initial convergence speed and the sensitivity to impulsive noise will increase with the 
threshold value $\xi$. The $R L S$ algorithm can be seen as a special kind of $R L M$ algorithm with a threshold $\xi=+\infty$.

Fig. 3 shows the tracking performance of the $R L M$ and $R L S$ algorithms for slowly varying signal amplitudes. In the simulation, the signal amplitudes were changed slowly as follows: $\alpha_{1}=1+0.0001 n$ and $\alpha_{2}=1.5+0.0001 n$. It can be seen that the $R L M$ algorithm is able to follow the change. However, the performance will degrade considerably if signal parameters are changing rapidly, due to the limitation of the signal model. The performance of the RLS algorithm is similar, except that it is significantly affected by the impulsive noise at time instants $[100,200$, and 350] as shown in Fig. 3. In addition, as the signal parameters are changing continuously with time, a smaller forgetting factor $\lambda_{a}$ should be used in order to "forget" data information in distant past. In other words, there is tradeoff between bias and variance of the estimated parameters.

\section{CONCLUSION}

A robust method for the recursive estimation of the frequencies and amplitudes of an exponential signal model under impulsive noise is presented. Based on the $M$ estimation, a recursive algorithm based on the recursive least $M$-estimate $(R L M)$ is developed. Simulation results show that the proposed method performs better than that based on the conventional least square method under impulsive noise environment. The algorithm also possesses low arithmetic complexity due to its recursive nature.

\section{REFERENCES}

[1] L. L. Scharf, Statistical Signal Processing: Detection, Estimation, and Time Series Analysis. Reading, Mass: Addison-Wesley, 1991.

[2] Y. Hua and T. K. Sarkar, "Matrix pencil method for estimating parameters of exponentially damped/ undamped sinusoids in noise," IEEE Trans. Acoustics, Speech, and Signal Processing, vol. 38, no.5, pp. 814-824, 1990.

[3] Y. Bresler and A. Macovski, "Exact maximum likelihood parameter estimation of superimposed exponential signals in noise", IEEE Trans. Acoust. Speech Signal Process., vol. ASSP-34, no. 5, pp. 1081-1089, 1986.

[4] P. J. Huber, Robust Statistics. New York: Wiley, 1981.

[5] Y. Wu and W. K. Tam, " $M$-estimation in exponential signal models", IEEE Transactions on Signal Processing, pp. 373-380, 2001.

[6] X. B. Wen and Z. Tian, "Bootstrap $M$-estimation in exponential signal models", IEE Electronics Letters, vol. 38, no. 23, pp. 1483-1485, 2002.

[7] J. G. Proakis, C. M. Rader, F. Ling and C. L. Nikias, Advanced Digital Signal Processing. New York: MacMillan, 1992.

[8] Y. Zou, S.C. Chan and T. S. Ng, "Least mean $M$-estimate algorithms for robust adaptive filtering in impulse noise", IEEE Trans. on Circuits and Systems-II-analog and digital signal processing, vol. 47, no. 12, pp. 1564-1569, 2000.

[9] Y. Zou, S. C. Chan, and T. S. Ng, "A recursive least $M$ estimate (RLM) adaptive filter for robust filtering in impulse noise," IEEE Signal Processing Letters, vol. 7, no. 11, pp. 324-326, 2000.

[10] S. C. Chan and Y. Zou, "A recursive least $M$-estimate algorithm for robust adaptive filtering in impulsive noise: fast algorithm and convergence performance analysis," to appear in IEEE Trans. Signal Processing.

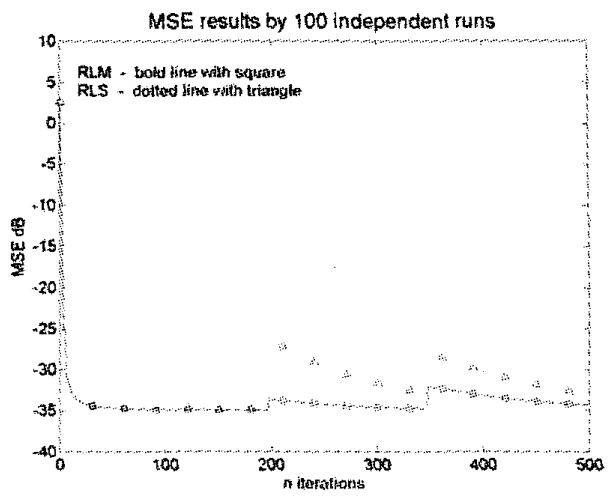

Fig. 1. The $M S E$ results of estimated signal $y$ using different algorithms: $R L M$ (bold line with square), and $R L S$ (dashed line with triangle). Threshold $\xi$ is chosen as $2.576 \cdot \sigma(e)$.

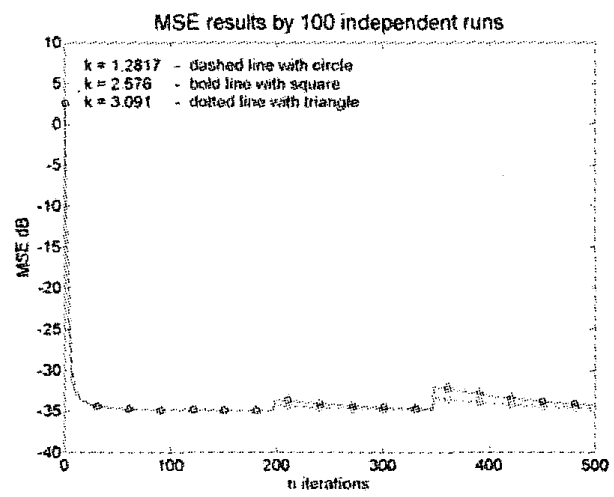

Fig. 2. The $M S E$ performance of estimated signal $y$ using $R L M$ with different threshold parameters $\xi=k \cdot \sigma(e): k=1.2817$ (dashed line with circle), $k=2.576$ (bold line with square), and $k=3.091$ (dotted line with triangle).

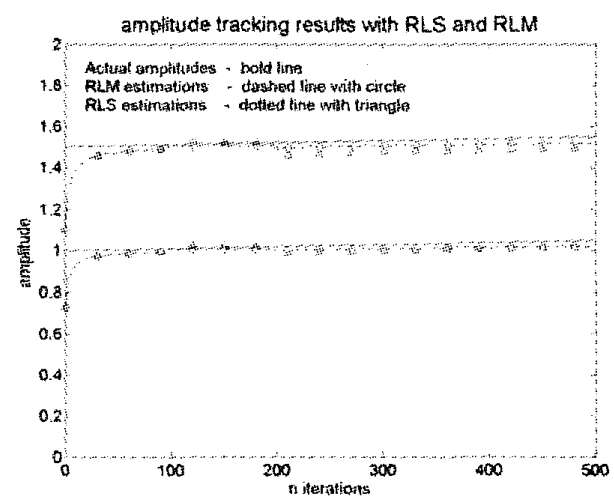

Fig. 3. Tracking amplitude change using $R L M$ (dashed line with circle) and $R L S$ (dotted line with triangle). The actual varying amplitudes are $\alpha_{1}=1+0.0001 n$ and $\alpha_{2}=1.5+0.0001 n$, indicated by bold line. Forgetting factor $\lambda_{a}$ is chosen as 0.9 . 